\title{
COMPARATIVE EFFECTS OF CAFFEINE \& L-THEANINE CONSUMPTION ON SUBJECTIVE CARDIOVASCULAR SIGNS AND NEUROPHYSIOLOGICAL RESPONSES.
}

\author{
Hira Zameer., Mehrunisa Iqbal., Lubna Anwer., Sadaf Ahmed \& Samia Mushtaq \\ 1. Department of Physiology, University of Karachi.
}

Corresponding author: hirashahkhan@hotmail.com

\begin{abstract}
Non-alcoholic beverages comprise of Caffeine and L-theanine as core ingredients. Caffeine is attributed to augment cardiovascular and neurophysiological responses by enhancing the neurotransmission of catecholamines after binding with adenosine receptors antagonistically. L-theanine, as a constituent of green tea is helpful in lowering blood pressure by antagonizing the effects of excitatory neurotransmitters after subsequent release of inhibitory neurotransmitter GABA. 87 healthy females with age 18-19 years, participated in the study were divided into three different groups. Group A was subjected to consume cold beverage (soft drink). Participants of group B \& C were allowed to take hot beverages in the form of tea and green tea respectively. Cardiovascular parameters and neurophysiological responses were assessed before and after 45 minutes of beverages intake. Statistical analysis revealed a significant improvement in the reaction time of tea and in concentration test of both tea and green tea consumers. In addition, a less significant increase in HR with tremendous decrease in PR was observed among green tea consumers. Furthermore, significant reduction in both systolic \& diastolic blood pressure was evident with green tea consumers.Experimental results are unveiling effectiveness of L-theanine over caffeine in lowering BP, HR \& PR after antagonizing the release of excitatory neurotransmitter along with the opening of ligand gated chloride channels that leads to hyperpolarization. Moreover, a favorable combination of caffeine \& L-theanine is responsible for enhancing cognitive performances, alertness and well being.
\end{abstract}

\section{KEYWORDS}

Unveiling, Hemodynamic, electroencephalographic, ingestion, neurophysiological, tolerance, cerebral infarction

\section{INTRODUCTION}

Regular intake based non-alcoholic hot and cold beverages comprise caffeine and theanine as their core ingredients. Having an average cup of tea that contains $40 \mathrm{mg}$ caffeine, peak plasma concentration of caffeine is achieved after 30 minutes of consumption. Caffeine easily crosses blood brain barrier and enhances the neurotransmission of epinephrine and norepinephrine selectively after binding with adenosine receptors antagonistically. Dietary components of tea have gained great considerations in relevance to their positive impacts on cognitive performance and psychological well-being. Demonstrations to psychopharmacological studies have revealed that tea, the most widely consumed beverage after water in the world is attributed to reduce the stress with wakeful relaxation and improves cognitive performances (Quinlan 2000; Hindmarch, 2000). Caffeine (1,3,7trimethylxanthine) is attributed to increase alertness and speeded reaction time (RT) predominantly (Fredholm, 1999; Smith, 2002), with profound reduction in physiological stress responses when compared with other caffeinated beverages (Steptoe, 2007). Hemodynamic parameters, such as blood pressure and heart rate can be influenced by the administration of methylxanthines. After entering into systemic circulation, methylxanthines have major impact on myocardial contractility \& conduction, vascular tone, and also on the sympathoadrenal system (Riksen, 2009). L-Theanine (gglutamylethylamide), also found in tea plant is a unique amino acid that have gained focus in the field of neuroscience having significant neuroprotective effects (Kakuda, 2002a). Moreover, electroencephalographic (EEG) recordings in humans revealed that Ltheanine consumption during resting state increased the activity of alpha frequency band (8-14 Hz) (Kobayashi, 1998; Juneja, 1999). In a dose dependent manner, L-theanine crosses the blood-brain barrier within 30 minutes of ingestion and thought to influence the release of neurotransmitters. Structurally, as L-theanine is similar to an excitatory neurotransmitter, glutamic acid (Nathan, 2006) so it

antagonizes the glutamate effects by inhibiting glutamate reuptake and blockade of glutamate receptors in the hippocampus (Kakuda,

2002) while increases the release and concentration of GABA along with decline in norepinephrine levels. Furthermore, L-theanine also contributes in lowering blood pressure (Eschenauer, 2006) after antagonizing caffeine effects and tone down the CNS responses. Furthermore, in response to a stressful task, L-theanine reduces heart rate and salivary immunoglobulin A by inhibiting excitation of cortical neurons (Kimura, 2007). Exposure to stress results in the activation of nervous system that further enhance the overwhelming release of vasoconstricting hormones that raise blood pressure (Kulkarni, 1998; Matthews, 2004) During this stressful response, consumption of Ltheanine suppresses raise in blood pressure by slowing down autonomic tone.

\section{MATERIALS \& METHODS}

The study was conducted over young adult females of university to evaluate the relative effects of regular based intake of regular cold and hot non-alcoholic beverages on subjective cardiovascular and neurophysiological responses. Prior to participation, an informed consent had been taken from the participants and experimental details were explained to the participants. 


\section{PARTICIPANTS}

87 young adult females, 18-19 years of age were participated in the study. Later, they were categorized into three groups. Group A volunteers $(n=46)$ were allowed to consume cold beverages in the form of soft drinks. Group B $(n=21)$ and C $(n=20)$ participants were instructed to consume hot beverages as tea and green tea respectively. Cardiovascular and neurophysiological responses were determined prior to and after 45 minutes of beverages consumption. Participants were requested to restrain from any kind food stuff except water for 3 hours before the experimental trials.

\section{NEUROPHYSIOLOGICAL RESPONSES}

\section{REACTION TIME TEST}

Fingertip reaction time of the participants was assessed before and after the consumption of beverages with the help of old manual "Drop Ruler Method" in neuroscience lab. Participants were asked to extend their right hands and hold out their thumb and forefingers. The 0 centimeter mark on the ruler was kept in between the subject's fingers. Later, they were allowed to catch the ruler with these two fingers when dropped. Point over the ruler was observed when it was caught by the participants. Distance was taken in centimeters later converted in milliseconds.

\section{CONCENTRATION TEST}

Volunteer's concentration was evaluated by "Einstein Distraction Index", a great technique to concentrate and avoid distraction for a specified period of time. Participants were provided with a blank piece of paper, with a phrase "I won't be distracted by that anymore" at the top of the paper. For about 5 minutes, they were given a passage for reading and instructed that every time if they were distracted, they were marked a check over that phrase, read that phrase silently and continue the reading again. After the passage of 5 minutes, checks marked the phrase were counted as the number of distractions that participants had in five minutes. All participants were provided with same passage for reading.

\section{CARDIOVASCULAR PARAMETERS}

Subjective cardiovascular signs of the participants such as blood pressure, heart rate and pulse were measured before and after the ingestion of beverages.

\section{RESULTS}

Statistically, data were analyzed by using Student's t test. As far as neurophysiological responses were concerned, improvement in reaction time test was noticeable among all participants. But the participants who assorted in group B had consumed caffeine in combination with L-theanine in the form of tea were found to be more focused and alerted towards the stimuli and come up with significant results $(\mathrm{P}<0.05)$. Concentration test of the participants was assessed by considering number of distractions during passage reading for about 5 minutes. L-theanine alone (green tea) and in combination with caffeine (tea) was proved to be more sufficient in enhancing concentration and focusing power of the individuals by reducing the distractions during a task at hand. After the consumption of tea (caffeine + L-theanine) and green tea (L-theanine), group B and C volunteers were evaluated to be more concentrated and deliberated during a passage reading task respectively $(\mathrm{P}<0.05)$ as compared to group $\mathrm{A}$ individuals $(\mathrm{P}=0.20)$ (Figure 1). Analysis of cardiovascular parameters with statistical tools clearly revealed that decline in systolic blood pressure was determined among each group participants, but a significant reduction in systolic blood pressure by 13 and $19 \mathrm{~mm} \mathrm{Hg}$ was evident amongst the candidates of group A and $\mathrm{C}$ who were assigned for the intake of soft drink (caffeine) and green tea (L-theanine) respectively $(\mathrm{P}<0.05)$ as compared to individuals who were belonged to tea (caffeine $+\mathrm{L}$ theanine) consumption group. Similarly, a considerable decline in diastolic blood pressure by $12 \mathrm{~mm} \mathrm{Hg}$ was more noticeable among the participants who were subjected to consume soft drink (caffeine) and green tea (L-theanine) $(\mathrm{P}<0.05)$ as compared to the group $\mathrm{B}$, tea consumers (Figure 2). Physiological rise in the heart rate of the individuals was observed when their basal heart rates were taken before and after consumption of hot and cold beverages. But a less significant rise in heart rate by $1 \mathrm{bpm}$ was remarkable among group $\mathrm{C}$ (L-theanine) candidates $(\mathrm{P}=0.31)$. In contrast to heart rate, a significant increase in pulse rate by $10 \mathrm{bpm}$ was reported within the members of group $\mathrm{C}$, green tea (L-theanine) consumers $(\mathrm{P}=0.01)$ (Figure 3).


Figure 1: Comparative effects of caffeine (soft drink), L-theanine (green tea) and caffeine + L-theanine (tea) were observed before and after the consumption of beverages with the help of reaction time and concentration test scores (Mean \pm S.E.). 

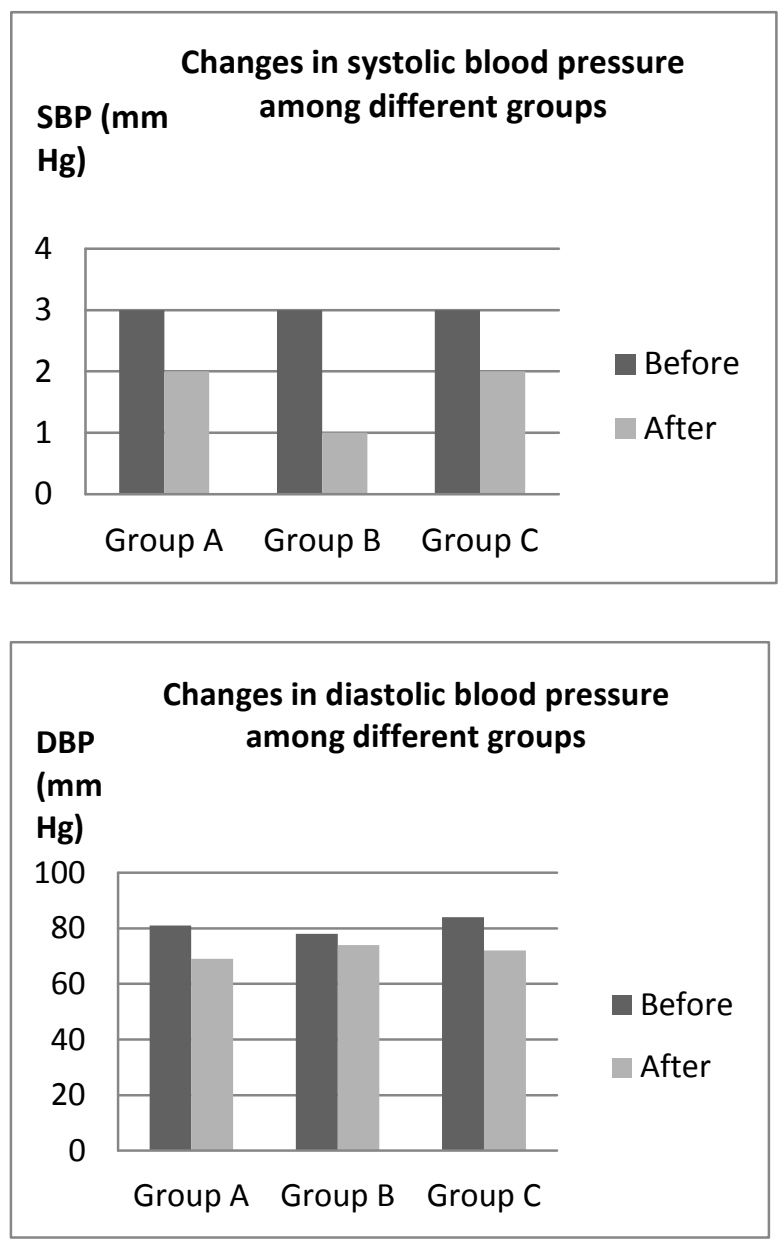

Figure 2: Expression of changes in systolic and diastolic blood pressure (Mean \pm S.E.) before and after the consumption of caffeine (soft drink), L-theanine (green tea) and caffeine + L-theanine (tea) among different group participants.

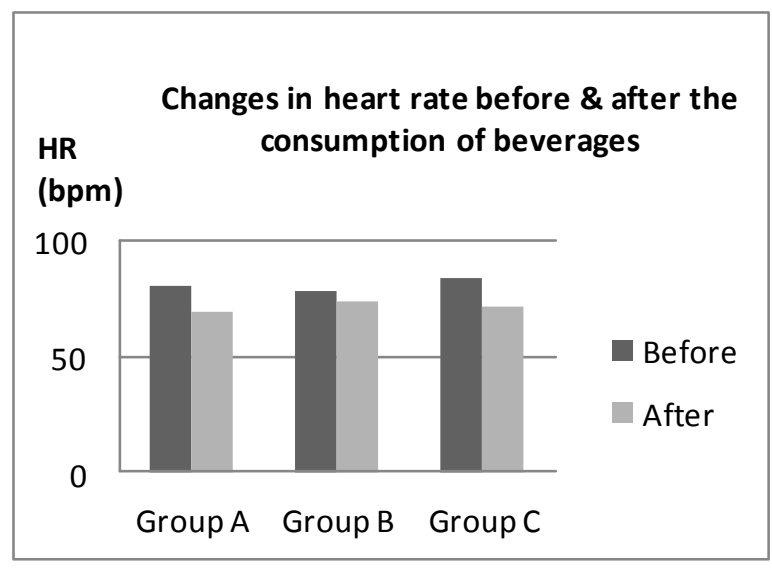

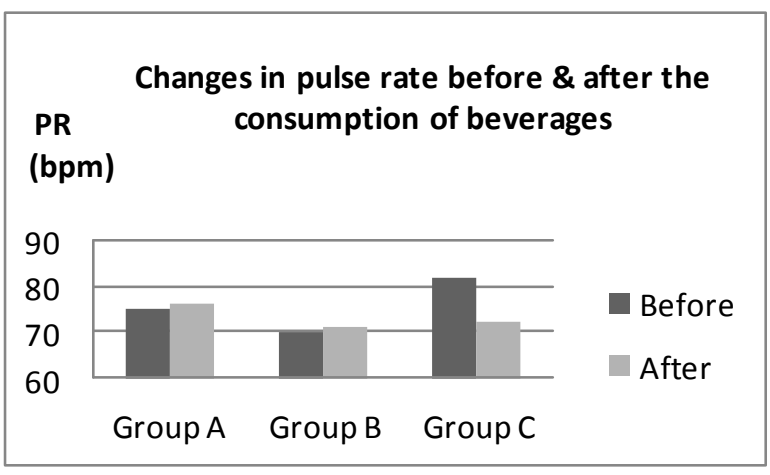

Figure 3: Expression of changes in heart rate and pulse rate (Mean \pm S.E.) of the candidates before and after the consumption of caffeine (soft drink), L-theanine (green tea) and caffeine + L-theanine (tea).

Table: Changes in Neurophysiological (RTT \& CT) and Cardiovascular parameters (SBP, DBP, HR \& PR)

\begin{tabular}{|c|c|c|c|c|c|c|}
\hline \multirow{2}{*}{$\begin{array}{c}\text { Param } \\
\text { eters }\end{array}$} & \multicolumn{2}{|c|}{ Group A $(\mathrm{n}=46)$} & \multicolumn{2}{c|}{ Group B $(\mathrm{n}=21)$} & \multicolumn{2}{c|}{ Group C (n=20) } \\
\cline { 2 - 7 } & Before & After & Before & After & Before & After \\
\hline RTT & $0.176 \pm$ & $0.170 \pm$ & $0.177 \pm$ & $0.159 \pm$ & $0.176 \pm$ & $0.168 \pm$ \\
& 0.001 & 0.002 & 0.002 & 0.003 & 0.002 & 0.003 \\
\hline CT & $2.54 \pm 0$ & $2.37 \pm 0$ & $2.57 \pm 0$ & $1.15 \pm 0$ & $2.65 \pm 0$ & $2.06 \pm 0$ \\
& .10 & .19 & .18 & .15 & .12 & .19 \\
\hline SBP & $119 \pm 1$. & $106 \pm 1$. & $114 \pm 2$. & $108 \pm 1$. & $124 \pm 3$. & $105 \pm 1$. \\
& 81 & 56 & 23 & 63 & 18 & 98 \\
\hline DBP & $81 \pm 1.6$ & $69 \pm 1.3$ & $78 \pm 2.2$ & $73 \pm 1.5$ & $84 \pm 3.0$ & $72 \pm 1.5$ \\
& 7 & 0 & 7 & 8 & 1 & 1 \\
\hline HR & $86 \pm 1.5$ & $95 \pm 2.0$ & $87 \pm 2.3$ & $94 \pm 3.4$ & $90 \pm 2.2$ & $91 \pm 2.3$ \\
& 7 & 3 & 7 & 6 & 9 & 7 \\
\hline PR & $75 \pm 2.2$ & $76 \pm 2.5$ & $70 \pm 2.2$ & $71 \pm 2.2$ & $82 \pm 4.0$ & $72 \pm 2.7$ \\
& 4 & 2 & 7 & 7 & & 1 \\
\hline
\end{tabular}

Abbreviations: RTT (reaction time test), CT (concentration test), SBP (systolic blood pressure), DBP (diastolic blood pressure), HR (heart rate) and PR (pulse rate)

\section{DISCUSSION}

The study was conducted to investigate the effects of non-alcoholic hot and cold beverages on subjective cardiovascular signs and neurophysiological responses of the participants. During a reaction time test, participants were found to be more alert and energetic after the consumption of caffeine and L-theanine containing beverages. Surprisingly, less time was taken by the participants of group B that consumed tea (caffeine + L-theanine) to react against a stimulus. As far as caffeine is concerned, low doses ( $35 \mathrm{mg}$ ) of particular stimulant has pronounced effects on cognitive performance in association with alertness, motivation, concentration and well being (Pandey, 2010). Moreover, during a concentration test, performance of group B (tea) and $\mathrm{C}$ (green tea) participants was sufficient with reduced number of distractions. Several studies support that during resting state, Ltheanine increases mental alertness by producing alpha waves bands letting the individuals' brain to be relaxed as well (Klein, 2007; Cahn, 2006; Montez, 2009). Oscillations and activity in the alpha-band has been linked with general attention processes (Montez, 2009). For example, when a human's brain focuses its attention in receiving information coming from one sensory modality (e.g. auditory) while trying to there is a very specific pattern of alpha activity when humans focus their attention on information coming from one sensory modality while excluding inputs coming from another distracting sensory modality (e.g. visual), a very specific pattern of alpha waves activity is 
noticeable (Swartz, 1998). Furthermore, Lipton Institute of Tea charged two randomized, double-blind crossover studies to investigate the effects of black tea on focused attention, by conducting standardized tests on alertness and subjective questionnaires. Results of the study revealed that L-theanine and caffeine, the two components of tea enhance the ability to focus and alertness after the consumption of 1-2 cups of black tea.During the determination of subjective cardiovascular signs, a tremendous decline in both systolic and diastolic blood pressure was observed after consuming soft drink (caffeine) and green tea (L-theanine) as compared to tea (caffeine + Ltheanine) ingestion. These findings reveal that beverages that are rich with either caffeine or L-theanine do not augment blood pressure whereas a beverage that comprise of both caffeine and L-theanine is less effective in reducing blood pressure. Numerous studies are come up with the finding that those individuals who do not consume caffeinated beverages on regular basis or recently withdraw caffeine intake, if caffeine is given to these individuals, a moderate rise in systolic ( 8 to $12 \mathrm{~mm} \mathrm{Hg}$ ) and diastolic ( 5 to $7 \mathrm{~mm} \mathrm{Hg}$ ) blood pressures will be observed that return to baseline values within 2 to 3 days because of the development of caffeine's tolerance. Furthermore, among the participants a significant increment in heart rate was manifested when caffeine was taken alone and in combination with $\mathrm{L}$ theanine as compare to ingestion of L-theanine only. Few experimental studies have revealed that exposure to any stressful stimuli causes caffeine to show similar physiological responses as human body makes homeostatic adjustments by raising plasma levels of the major stress hormones, including epinephrine, norepinephrine and cortisol leading to the activation of both components of neuroendocrine stress responses such as sympathetic adrenal-medullary and the hypothalamic pituitary adrenal-cortical. In the end, when caffeinated beverages are taken in everyday life, caffeine starts to act like a stressor itself (James, 2002). In our study, as participants were already known to details of the experiments so they might felt stressful against reaction time and concentration test that were assessed after noting down individuals' cardiovascular parameters. In contrast to heart rate, a less prominent increment was observed pulse rate when caffeine was taken alone and in combination with L-theanine. Surprisingly, a significant decline was examined when L-theanine was taken alone in the form of green tea. Findings of our study highlight a recent prospective cohort study in which a significant reduction in mortality due to cardiovascular diseases (26\%), stroke (37\%) and cerebral infarction (51\%) was observed in 240,530 Japanese Adults that consumed 5 or more cups of green tea per day. In the end, according to a research that has been published in a recent issue of the American Journal of Cardiology, although people who drank green tea everyday were no less likely to have coronary artery disease than people who did not drink tea, they were much less likely to have a heart attack.( Kuriyama, 2006).In last, due to less numbers of young adults males we decided to do not include the data of male participants. The parameters that were examined in female participants might be evaluated with different findings in male volunteers. In future, we would like to reproduce the study with male candidates along with gender comparsion.

\section{CONCLUSION}

Our research study suggested that caffeine in combination with Ltheanine improves cognitive performance and selective attention. Furthermore, helps to avoid distracting stimuli by letting brain to be more concentrated and alert. In addition, consumption of caffeine and L-theanine can be a preventive approach in reducing cardiovascular risks.

\section{REFERENCES}

- Quinlan, P. T., Lane, J., Moore, K. L., Aspen, J., Rycroft, J. A., \& O'Brien, D. C. (2000). The acute physiological and mood effects of tea and coffee: the role of caffeine level. Pharmacology, biochemistry, and behavior, 66(1), 19.
- Hindmarch, I., Rigney, U., Stanley, N., Quinlan, P., Rycroft, J., \& Lane, J. (2000). A naturalistic investigation of the effects of daylong consumption of tea, coffee and water on alertness, sleep onset and sleep quality.Psychopharmacology, 149(3), 203-216.

- Fredholm, B. B., Bättig, K., Holmén, J., Nehlig, A., \& Zvartau, E. E. (1999). Actions of caffeine in the brain with special reference to factors that contribute to its widespread use. Pharmacological reviews, 51(1), 83-133.

- Smith A. (2002) Effects of caffeine on human behaviour. Food Chem Toxicol.;40:1243-55.

- Steptoe, A., Gibson, E. L., Vounonvirta, R., Williams, E. D., Hamer, M., Rycroft, J. A., ... \& Wardle, J. (2007). The effects of tea on psychophysiological stress responsivity and post-stress recovery: a randomised double-blind trial.Psychopharmacology, 190(1), 81-89.

- Riksen, N. P., Rongen, G. A., \& Smits, P. (2009). Acute and longterm cardiovascular effects of coffee: implications for coronary heart disease.Pharmacology and therapeutics, 121(2), 185-191.

- Kakuda, T., (2002) Neuroprotective effects of the green tea components L-theanine and catechins. Biol Pharm Bull.25:1513-8.

- Kobayashi, K., Nagata, Y., Aloi, N., Juneja, L., Kim, M., Yamamoto, T., Sugimoto, S.,(1998) Effects of L-theanine on the release of a-brain waves in human volunteers. Nihon Nogeikagaku Kaishi. 72:153-7.

- Juneja, L. R., Chu, D. C., Okubo, T., Nagato, Y., \& Yokogoshi, H. (1999). L-theanine - a unique amino acid of green tea and its relaxation effect in humans.Trends in Food Science \& Technology, 10(6), 199-204.

- Nathan, P. J., Lu, K., Gray, M., \& Oliver, C. (2006). The Neuropharmacology of L-Theanine (N-Ethyl-L-Glutamine) A Possible Neuroprotective and Cognitive Enhancing Agent. Journal of Herbal Pharmacotherapy, 6(2), 21-30.

- Kakuda, T., Nozawa, A., Sugimoto, A., \& Niino, H. (2002). Inhibition by theanine of binding of $[3 \mathrm{H}]$ AMPA, $[3 \mathrm{H}]$ kainate, and [3H] MDL 105,519 to glutamate receptors. Bioscience, biotechnology, and biochemistry, 66(12), 2683-2686.

- Eschenauer, G., \& Sweet, B. V. (2006). Pharmacology and therapeutic uses of theanine. American journal of health-system pharmacy: AJHP: official journal of the American Society of Health-System Pharmacists, 63(1), 26-28.

- Kimura, K., Ozeki, M., Juneja, L. R., \& Ohira, H. (2007). LTheanine reduces psychological and physiological stress responses. Biological psychology, 74(1), 39-45.

- Kulkarni, S., O'Farrell, I., Erasi, M., \& Kochar, M. S. (1998). Stress and hypertension. WMJ: official publication of the State Medical Society of Wisconsin, 97(11), 34.

- Matthews, K. A., Katholi, C. R., McCreath, H., Whooley, M. A., Williams, D. R., Zhu, S., \& Markovitz, J. H. (2004). Blood pressure reactivity to psychological stress predicts hypertension in the CARDIA study. Circulation,110(1), 74-78.

- Pandey, S.(2010) Tea: Effects on cardiovascular system, blood pressure, EEG and many other system of human being.

- Klein, S., \& Thorne, B., (2007) Biological psychology. New York, N.Y.: Worth.

- Cahn, B. R., \& Polich, J. (2006). Meditation states and traits: EEG, ERP, and neuroimaging studies. Psychological bulletin, 132(2), 180.

- Montez, T., Poil, S. S., Jones, B. F., Manshanden, I., Verbunt, J. P., van Dijk, B. W., ... \& Linkenkaer-Hansen, K. (2009). Altered temporal correlations in parietal alpha and prefrontal theta oscillations in early-stage Alzheimer disease.Proceedings of the National Academy of Sciences, 106(5), 1614-1619.

- Swartz, B. E., \& Goldensohn, E. S. (1998). Timeline of the history of EEG and associated fields. Electroencephalography and clinical Neurophysiology, 106(2), 173-176.

- Lane, J. D., Pieper, C. F., Phillips-Bute, B. G., Bryant, J. E., \& Kuhn, C. M. (2002). Caffeine affects cardiovascular and 
neuroendocrine activation at work and home. Psychosomatic medicine, 64(4), 595-603.

- Kuriyama, S., Shimazu, T., Ohmori, K., Kikuchi, N., Nakaya, N., Nishino, Y., \& Tsuji, I. (2006). Green tea consumption and mortality due to cardiovascular disease, cancer, and all causes in Japan. JAMA: the journal of the American Medical Association, 296(10), 1255-1265. 